\title{
BRCA1 and BRCA2 Common Mutations in Iranian Breast Cancer Patients: a Meta Analysis
}

\author{
Mohammad Forat-Yazdi ${ }^{1}$, Hossein Neamatzadeh ${ }^{2 *}$, Mohammad Hasan \\ Sheikhha $^{2}$, Masoud Zare-Shehneh ${ }^{2}$, Mortaza Fattahi ${ }^{3}$
}

\begin{abstract}
Background: To date several common mutations in BRCA1 and BRCA2 associated with breast cancer have been reported in different populations. However, the common BRCA1 and BRCA2 mutations among breast cancer patients in Iran have not been described in detail. Materials and Methods: To comprehensively assess the frequency and distribution of the most common BRCA1 and BRCA2 mutations in Iranian breast cancer patients, we conducted this meta-analysis on 13 relevant published studies indentified in a literature search on PubMed and SID. Results: A total of 11 BRCA1 and BRCA2 distinct common mutations were identified, reported twice or more in the articles, of which $10(c .2311 \mathrm{~T}>\mathrm{C}, \mathrm{c.3113} \mathrm{A}>\mathrm{G}, \mathrm{c.4308 \textrm {T }}>\mathrm{C}, \mathrm{c} .4837 \mathrm{~A}>\mathrm{G}, \mathrm{c} .2612 \mathrm{C}>\mathrm{T}$, c.3119G $>A$, c.3548A >G, c.5213G $>$ A c.IVS16-92A/G, and c.IVS16-68A/G) mutations were in BRCA1, and 1 (c.4770A>G) was in BRCA2. The mutations were in exon 11, exon 13, intron 16, and exon 20 of BRCA1 and exon 11 of BRCA2. All have been previously reported in different populations. Conclusions: These meta analysis results should be helpful in understanding the possibility of any first true founder mutation of BRCA1/BRCA2 in the Iranian population. In addition, they will be of significance for diagnostic testing, genetic counseling and for epidemiological studies.
\end{abstract}

Keywords: Breast cancer - BRCA1 - BRCA2 - common mutations - Iran

Asian Pac J Cancer Prev, 16 (3), 1219-1224

\section{Introduction}

Breast cancer is the most common cause of mortality among women accounting for $23 \%$ all cancers (Perez et al., 2009). In recent years, the mortality rate from breast cancer has increased rapidly in all countries. Breast cancer is the most prevalent cancer in Iranian women with an increase in incidence rates in recent years. In Iran, even though the prevalence of breast cancer is lower compared to western countries, it is the most common malignancy among women (Rezaianzadeh et al., 2011). There is an increasing trend for breast cancer mortality in Iran during 1995 to 2004 from 1.40 to 3.52 per 100,000 (Taghavi et al., 2012). In the next decades, Iran will face an upsurge in the incidence of the disease (Dey and Soliman, 2010).

Breast-ovarian cancer (BOC)-causing mutations and other genetic variants are distributed along the entire coding and non-coding regions of BRCA1 and BRCA2, and more than 3400 gene variants have been described in the Breast Cancer Information Core (Solano et al., 2012). New variants continue to be detected worldwide, mostly in BRCA1. Individuals with an inherited inactivating mutation in BRCA1 (MIM\# 113705) or BRCA2 (MIM\# 600185) have an increased risk of developing early-onset breast and ovarian cancers (Welcsh and King et al., 2001). Inherited mutations in BRCA 1 account for $40-45 \%$ of all hereditary BC cases, but approximately $80 \%$ of cases in families with multiple cases of breast and ovarian cancers (Easton et al., 1999).

The aim of this study was to survey the spectrum of most common mutations among Iranian breast cancer patients.

\section{Materials and Methods}

\section{Search strategy}

The electronic databases PubMed, EMBASE, and Scientific Information Database (SID) were searched up to October 30, 2014. The final search strategy used for each database was based on keywords "BRCA1," "BRCA2," "Iran," "Breast cancer," and "polymorphism." Synonyms and different styles of the search terms were also used in the search in order to obtain every relevant paper. Casecontrol studies containing available BRCA1 and BRCA2 
genes mutations frequencies were chosen. Only research articles were included and the language was not limited. Reference lists of the included studies on related topics were also screened for additional studies.

\section{Data extraction}

Data were carefully and independently extracted from the relevant papers by two of the authors (HN and MZS) using the same standardized form. The following data were collected from each study: first author, year of publication, source of controls, and genotype distribution. In case of disagreement, a third reviewer assessed the articles until an agreement was reached. The following items were collected from each article: first author, publication year, country or

\section{Statistical analysis}

The strength of association between common mutations in BRCA1 and BRCA 2 and breast cancer risk was assessed for each study by frequency by using of crude ORs with 95\%CIs. For all studies, only the frequency of mutation which repeatedly reported twice or more in different articles was evaluated. Frequency of each BRCA mutation carriers was more than $1 \%$ in meta-analyses by using the number of patients with event mutation and the total number of patients. The statistical heterogeneity among studies was assessed with the $\mathrm{I} 2$ test was used to quantify inconsistency. An I 2 value $\geq 50 \%$ was considered to represent significant statistical heterogeneity. The fixedeffects model (the Mantel-Haenszel method) was used to calculate the pooled OR with $95 \% \mathrm{CI}$; Otherwise we applied the random effects model (the DerSimonian and Laird method) (Begg et al., 1994; Egger et al., 1997). These two models provided similar results when between studies heterogeneity was absent. Funnel plots and the linear regression asymmetry test by Egger et al., were used to evaluate potential publication bias (Egger et al., 1997).All statistical analyses were performed in Statistical Analysis System software (v.9.1.3; SAS Institute, Cary, NC) and Review Manage (v.4.2; Oxford, England), using two-sided $\mathrm{P}$ values.

\section{Results}

\section{Study characteristics}

Our systematic literature search identified 22 studies that met the inclusion criteria. After deduplication and exclusion of the clearly irrelevant studies, we eventually included 13 studies (Bar-Sade et al., 1998; Ghaderi et al., 2001; Yassaee et al., 2002; Moslehi et al., 2003; Pietschmann et al., 2005; Quintana-Murci et al., 2005; Mehdipour et al., 2006; Rassi et al., 2008; Fattahi et al., 2009; Saleh gohari et al., 2012, Keshavarzi et al., 2012; Keshavarzi et al., 2012; and Kooshyar et al., 2013) involving 1183 breast cancer patients. Figure 1 shows the study selection process.

Baseline characteristics of the 13 studies are summarized a synopsis of the included studies, briefly describing study population, mutation scanning method, mutation carriers information, and the enrollment criteria of families and subjects (Tables 1,2). The 13 studies included report on 7 different screening methods for the detection of BRCA1 and BRCA2 mutations: In these

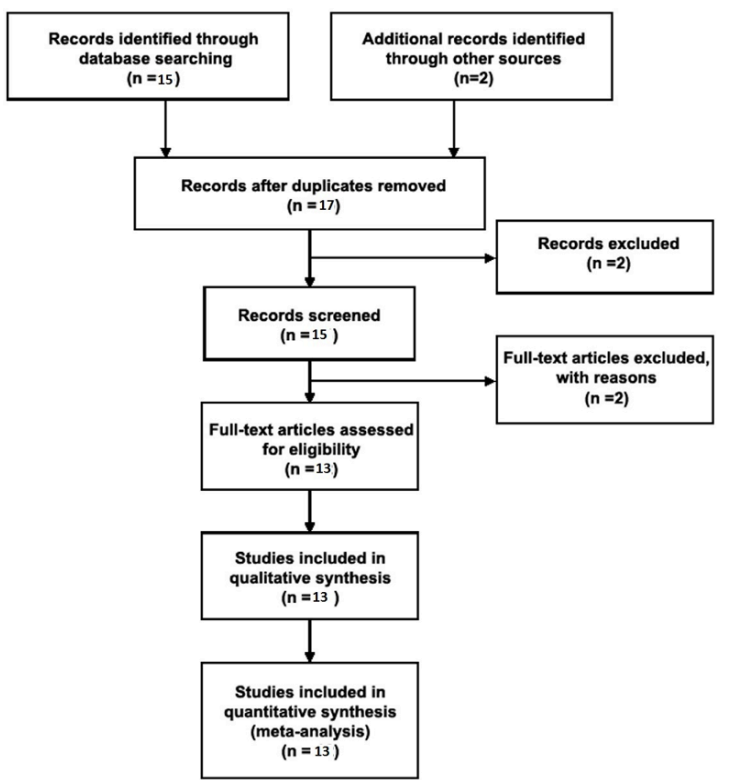

Figure 1. Flow Diagram of the Study Selection Process

Table 1. Characteristics of All 13 Studies Retrieved with the Searching Strategy for BRCA1

\begin{tabular}{|c|c|c|c|c|c|}
\hline \multirow[t]{2}{*}{ Author } & \multirow[t]{2}{*}{ Year } & \multicolumn{2}{|c|}{ Study population } & \multirow[t]{2}{*}{ Technique } & \multirow[t]{2}{*}{ Exo/Int } \\
\hline & & NO. & $\operatorname{Age}(y r)$ & & \\
\hline Bar-Sade et al & 1998 & 150 Iranian Jews & 37 & HA/Direct sequencing & 2 \\
\hline Ghaderi et al & 2001 & 22(BC) 18 (control) & 42 & Direct sequencing & $\begin{array}{l}\text { 11-2, 11-3a, 11-3b, 11-4, } \\
\text { 13,16,IVS16,IVS16,IVS18 }\end{array}$ \\
\hline Yassaee et al & 2002 & 83 & $<45$ & PTT, SSCP/HP & $2,11,20$ \\
\hline Moslehi et al & 2003 & 1 Family with 4 cases (HOBS) & 56 & SSCP-PCR & \\
\hline Murci et al & 2005 & 442 (only males) & $45-67$ & RFLP-PCR & 2 \\
\hline Pietschmann et al & 2005 & 10 high risk breast cancer families & $<50$ & Direct sequencing & $16,17,18,24$ \\
\hline Mehdipour et al & 2006 & 396(Female), 4(Male) & $48.8 \pm 11.3$ & PCR & 2 \\
\hline Rassi et al & 2008 & 16(FBC), 18(NFBC) & $15-95,25-80$ & Multiplex-PCR & 2 \\
\hline Fattahi et al & 2009 & 250 (SBC), 55(FBC), 200(HF) & $\begin{array}{l}45.1 \pm 9.2 \\
32.0 \pm 7.3\end{array}$ & Multiplex-PCR & 2 \\
\hline Keshavarzi et al & 2011 & 27 (BC), $50(\mathrm{HF})$ & $\leq 35$ & Direct sequencing & $7,9,11,13,16,20$ \\
\hline Saleh-gohari et al & 2012 & 22 (female), 8 (male) & 51 & Direct sequencing & $2,11,11$ \\
\hline Keshavarzi et al & 2012 & 36 (FBC), 49 (NFBC), 61 (Control) & $\leq 35$ & & $\begin{array}{l}\text { IVS1, IVS1, 2, IVS2, } \\
\text { IVS5, 6, 11, 13, 16, } 20\end{array}$ \\
\hline Kooshyar et al & 2013 & $\begin{array}{l}39(\mathrm{BC}) \\
29(\mathrm{HR})\end{array}$ & $\begin{array}{c}49.3 \\
23-72\end{array}$ & SSCP-PCR & 2,11 \\
\hline
\end{tabular}


Table 2. Characteristics of All 13 Studies Retrieved with the Searching Strategy for BRCA2

\begin{tabular}{|c|c|c|c|c|c|}
\hline \multirow[t]{2}{*}{$\overline{\text { Author }}$} & \multirow[t]{2}{*}{ Year } & \multicolumn{2}{|c|}{ Study population } & \multirow[t]{2}{*}{ Technique } & \multirow{2}{*}{ Exo/Int } \\
\hline & & NO. & $\operatorname{Age}(\mathrm{yr})$ & & \\
\hline Yassaee et al & 2002 & 83 & $<45$ & PTT, DS, SSCP/HA & $11,17,18,23$ \\
\hline Moslehi et al & 2003 & $\begin{array}{l}1 \text { Family with } 4 \\
\text { cases (HOBS) }\end{array}$ & $\begin{array}{c}56 \\
45-67\end{array}$ & PTT & 11 \\
\hline Pietschmann et al & 2005 & $\begin{array}{l}20 \\
250(\mathrm{SBC})\end{array}$ & $\begin{array}{c}50 \\
45.1 \pm 9.2\end{array}$ & $\begin{array}{l}\text { Direct sequencing } \\
\text { Multiplex-PCR }\end{array}$ & $2,8,10,11,14,16,21$ \\
\hline Fattahi et al & 2009 & $\begin{array}{l}55(\mathrm{FBC}) \\
200(\mathrm{HF})\end{array}$ & $32.0 \pm 7.3$ & & \\
\hline keshavarzi et al & 2011 & $\begin{array}{l}27(\mathrm{BC}) \\
50(\mathrm{HF})\end{array}$ & $\leq 35$ & Direct sequencing & 10,11 \\
\hline keshavarzi et al & 2012 & $\begin{array}{l}36 \text { (FBC) } \\
49 \text { (NFBC) } \\
61 \text { (Control) }\end{array}$ & $\leq 35$ & Direct sequencing & IVS6, 11 \\
\hline
\end{tabular}

Table 3. BRCA1 and BRCA2 Common Mutations Identified in 13 Studies

\begin{tabular}{|c|c|c|c|c|c|}
\hline $\begin{array}{l}\text { BRCA } \\
\text { mutation }\end{array}$ & $\begin{array}{l}\text { Exon } \\
\text { /intron }\end{array}$ & $\begin{array}{c}\text { Mutation } \\
\text { effect }\end{array}$ & $\mathrm{n} / \mathrm{Na}$ & Frequency & References \\
\hline \multicolumn{6}{|l|}{ BRCA1 } \\
\hline c. $2311 \mathrm{~T}>\mathrm{C}$ & 11 & Synonymous & $17 / 154$ & 0.131 & $\begin{array}{l}\text { Ghaderi et al., 2001; Pietschmann et al., 2005; Keshavarzi et al., 2011; } \\
\text { Keshavarzi et al., } 2012\end{array}$ \\
\hline c. $2612 \mathrm{C}>\mathrm{T}$ & 11 & Misssense & $18 / 69$ & 0.269 & Ghaderi et al., 2001; Pietschmann et al., 2005; Keshavarzi et al., 2011 \\
\hline c. $3113 \mathrm{~A}>\mathrm{G}$ & 11 & Misssense & $35 / 105$ & 0.334 & $\begin{array}{l}\text { Ghaderi et al., 2001; Pietschmann et al., 2005; Keshavarzi et al., 2011; } \\
\text { Keshavarzi et al., } 2012\end{array}$ \\
\hline c. $3119 \mathrm{G}>\mathrm{A}$ & 11 & Misssense & $26 / 132$ & 0.202 & Ghaderi et al., 2001; Pietschmann et al., 2005; Keshavarzi et al., 2011 \\
\hline c. $3548 \mathrm{~A}>\mathrm{G}$ & 11 & Misssense & Oct-42 & 0.243 & Ghaderi et al., 2001; Pietschmann et al., 2005 \\
\hline c. $4308 \mathrm{~T}>\mathrm{C}$ & 13 & Synonymous & $26 / 154$ & 0.175 & $\begin{array}{l}\text { Ghaderi et al., 2001; Pietschmann et al., 2005; Keshavarzi et al., 2011; } \\
\text { Keshavarzi et al., } 2012\end{array}$ \\
\hline c. $4837 \mathrm{~A}>\mathrm{G}$ & 16 & Misssense & $30 / 154$ & 0.201 & $\begin{array}{l}\text { Ghaderi et al., 2001; Pietschmann et al., 2005; Keshavarzi et al., 2011; } \\
\text { Keshavarzi et al., } 2012\end{array}$ \\
\hline IVS16-92A/G & 16 & Unknown & $6 / 42$ & 0.147 & Ghaderi et al., 2001; Pietschmann et al., 2005; \\
\hline IVS16-68A/G & 16 & Unknown & $9 / 44$ & 0.219 & Ghaderi et al., 2001; Pietschmann et al., 2005 \\
\hline c. $5213 \mathrm{G}>\mathrm{A}$ & 20 & Misssense & $9 / 112$ & 0.096 & Keshavarzi et al., 2011; Keshavarzi et al., 2012 \\
\hline \multicolumn{6}{|l|}{ BRCA2 } \\
\hline 4075delGT & 11 & Synonymous & $5 / 122$ & 0.045 & Keshavarzi et al., 2011; Keshavarzi et al., 2012 \\
\hline
\end{tabular}

${ }^{\mathrm{a}} \mathrm{n}$ referred to the number of patients with event mutation, $\mathrm{N}$ referred to the total number of patients in total families; ${ }^{\mathrm{b}}$ These data were calculated through cumulative outcomes from all related articles; ${ }^{\mathrm{c}}$ These frequencies were obtained from meta-analysis results from all related articles

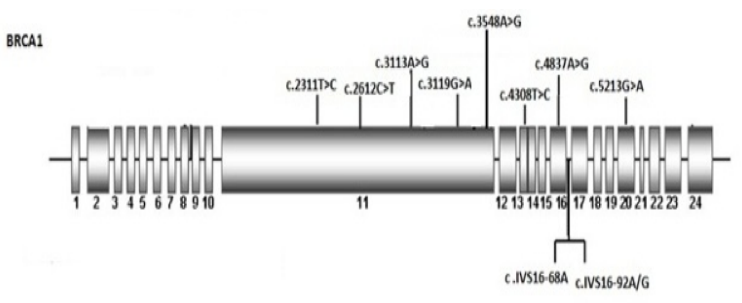

Figure 2. Position of Common Mutations with in BRCA1 in Iranian BC Patients

methods, Direct sequencing was more than one evaluation study identified and the distribution of these screening methods was as follows: 9 DHPLC, 7 SSCP, 6 PTT, 4 HA, 2 DGGE, 2 CSGE and 4 studies conducted sequencing to confirm each germline BRCA1/2 variant.

As shown in Figure 2, 11 common mutations were identified from all 13 studies, at exon 11, exon 13, intron 16 , and exon 20 of BRCA1 and exon 11 of BRCA2.

To evaluate the frequency of 11 common mutations which repeatedly reported twice or more in different articles, we conducted meta-analysis and the results were shown in Table 1. For those 11 mutations, 4 studies had repeatedly reported the c.2311T $>$ C, c.3113A, c.4308T $>$ C, c. $4837 \mathrm{~A}>\mathrm{G}$, and c.2311T>C, 3 studies had repeatedly reported the c. $2612 \mathrm{C}>\mathrm{T}$, and c. $3119 \mathrm{G}>\mathrm{A}$, and 2 studies had repeatedly reported the c.3548A $>$ G, c.IVS16-92A/G, c.IVS16-68A/G, and c.5213G $>$ A. For those mutations which were not repeatedly reported in all 13 studies, we only selectively listed the common mutations (frequency more than 5\%) in our study. For the BRCA1 (Figure 3 ), after conducting meta-analysis, we found that the overall frequency of c.3113A $>\mathrm{G}$ was 0.3 (95\%CI $0.24-$ $0.44, p=0.00$ for heterogeneity test), the frequency of c. $2311 \mathrm{~T}>\mathrm{C}$ was $0.13(95 \% \mathrm{CI} 0.08-0.20, \mathrm{p}=0.00$ for heterogeneity test), the frequency of c. $2612 \mathrm{C}>\mathrm{T}$ was 0.27 (95\% CI $0.17-0.57, \mathrm{p}=0.00$ for heterogeneity test), the frequency of c.3548A $>\mathrm{G}$ was 0.24 (95\% CI 0.01 $0.13, \mathrm{p}=0.00$ for heterogeneity test), the frequency of c. $4308 \mathrm{~T}>\mathrm{C}$ was 0.17 (95\%CI $0.12-0.24, \mathrm{p}=0.00$ for heterogeneity test), the frequency of c. $4837 \mathrm{~A}>\mathrm{G}$ was 0.20 (95\% CI $0.14-0.27, \mathrm{p}=0.00$ for heterogeneity test), the frequency of c.IVS $16-92 \mathrm{~A} / \mathrm{G}$ was 0.22 (95\%CI 0.10 $0.40, \mathrm{p}=0.00$ for heterogeneity test), the frequency of c.IVS16-68A/G was 0.22 (95\%CI $0.12-0.38, p=0.00$ for heterogeneity test), the frequency of c. $3119 \mathrm{G}>\mathrm{A}$ was 0.20 (95\% CI $0.14-0.28, p=0.00$ for heterogeneity test), and the 


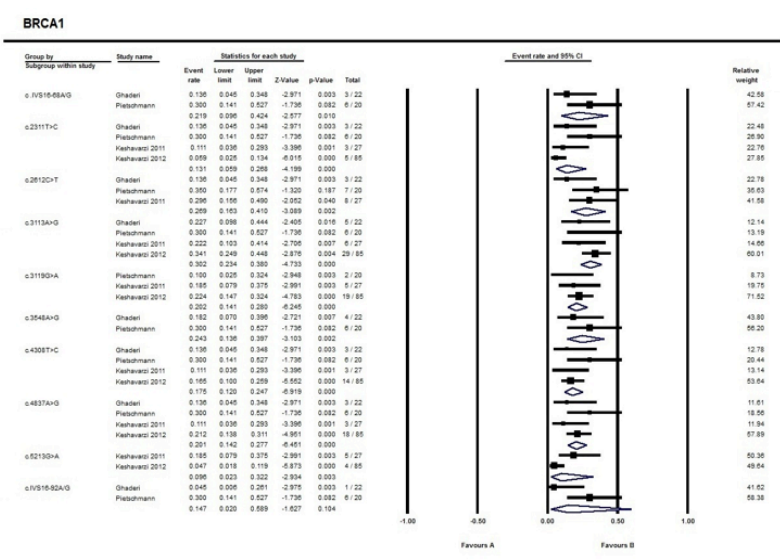

Figure 3. Forest Plot Showing the Odds Ratio of Each Study and the Pooled Estimate in BRCA1

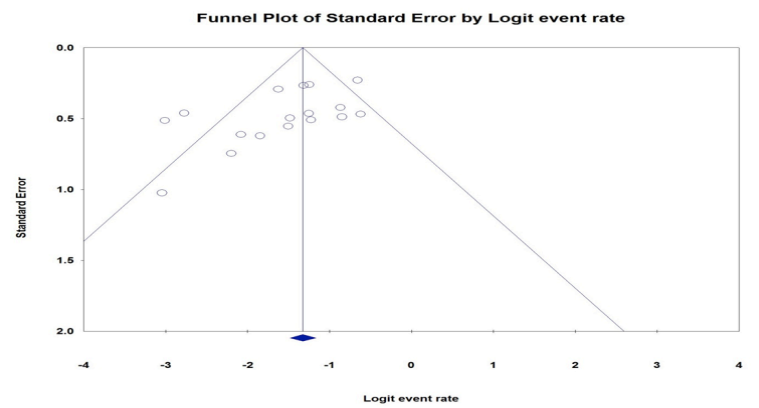

Figure 4. Funnel Plot of Standard Error by Log Odds Ratio Showing Analysis of Publication Bias of Studies in Spontaneously Aborted Embryos

frequency of c.5213G $>\mathrm{A}$ was 0.09 (95\% CI $0.05-0.17$, $\mathrm{p}=0.00$ for heterogeneity test).

For the BRCA2, the overall frequency of $4075 \mathrm{delGT}$ was 0.02 (95\%CI $0.019-0.103, \mathrm{p}=0.00$ for heterogeneity test).

\section{Publication bias}

Egger test for intercept showed no evidence of publication bias (intercept $=-1.85 ; \mathrm{SE}=0.67 ; 95 \% \mathrm{CI}$ $=-3.23-0.47$; P2-tailed = 0.01). Classic Fail-Safe "N"= 4 , missing from the analysis for every published study included, which further supports absence of publication bias. A funnel plot of SE by log OR was generated. Almost symmetric distribution of studies on the funnel plot shows absence of publication bias (Figure 4).

\section{Discussion}

To date, no reports have been published about BRCA1 and BRCA2 most common mutations in the Iranian population. To determine frequent mutations within the Iranian population, we analyzed together the other 13 studies for this population and calculated pooled mutation frequencies. We believe that this Meta-analysis result will be of significance both for diagnostic testing and for epidemiological studies. BRCA1 and BRCA2 are both large genes, and complete analysis of the coding regions is expensive and time-consuming. The identification of recurrent mutations allows for the rapid diagnosis of BRCA1-mutation carriers in Iranian individuals with relatively high sensitivity.
The majority of these 11 mutations described here are frequent in other populations. Recently, Medimegh et al., found that c. $2082 \mathrm{C}>\mathrm{T}, \mathrm{c} .3113 \mathrm{~A}>\mathrm{G}, \mathrm{c} .3119 \mathrm{G}>\mathrm{A}$, c.3548A $>\mathrm{G}$ and c.4837A $>\mathrm{G}$ SNPs were not associated with breast cancer disease (FBC or SBC) with $\mathrm{P}$ value $>0.05$.

The most commonly observed mutation in the present study was the c.3113A $>\mathrm{G}$, which accounted for $29.8 \%$ of all common mutations. This mutation has previously been reported in various ethnic groups. However, Medimegh et al., have found c.3113A $>\mathrm{G}$ was not associated with breast cancer disease (Medimegh et al., 2014). In addition, Dombernowsky et al., in a large study, evaluated risk associated of breast and/or ovarian cancer by 9 missense polymorphisms in BRCA1 c.1067A $>\mathrm{G}$, c. $2612 \mathrm{C}>\mathrm{T}$, c. $3113 \mathrm{~A}>\mathrm{G}$, c. $4837 \mathrm{~A}>\mathrm{G}$, c. $4956 \mathrm{G}>\mathrm{A}$ and $\mathrm{BRCA} 2$ c. $865 \mathrm{~A}>\mathrm{C}$, c. $1114 \mathrm{~A}>\mathrm{C}, \mathrm{c} .4258 \mathrm{G}>\mathrm{T}$, and c. $5744 \mathrm{C}>\mathrm{T}$. They found no association between heterozygosity or homozygosity for any of the nine polymorphisms and risk of breast and/or ovarian cancer in either study (Dombernowsky et al., 2009).

Medimegh et al., Wild-type alleles and genotypes of c. $442-58$ delT, c.2311T $>$ C, c. $2612 \mathrm{C}>\mathrm{T}$ and c.4308T $>\mathrm{C}$ are clearly associated with familial breast cancer with an odds ratio ranging from 2.49 to 4.66 . They found that among the four associated SNPs to familial breast cancer, the $\mathrm{c} .2612 \mathrm{C}>\mathrm{T}$ variant could have an effect on the protein sequence with an amino acid change (Proline to Leucine) at position 871, suggesting an alteration on the protein function and an ambivalent role of wild allele to familial breast cancer susceptibility. However, Dombernowsky et al., found no association between c. $2612 \mathrm{C}>\mathrm{T}$ and breast cancer (Dombernowsky et al., 2009).

The BRCA1 c.2311T>C missense mutation in exon 11 accounted for $4.5 \%$ of families with mutations. This mutation has previously been reported in various ethnic groups. Combination between TT genotype of c.2311T[C and miR-1179 over-expression could generate a lack of BRCA1 protein leading to a high risk of familial breast cancer with distant metastases.

Pilato et al. studied both transmission of BRCA1 and BRCA2 pathogenic mutations and polymorphic variants in breast cancer familial members. They found that SNPs BRCA1 c.3548A>G (p.Lys1183Arg) were more frequently present in breast cancer relatives belonging to families tested negative for BRCA1 and BRCA2 mutations (Pilato et al., 2011). Also, Cherbala et al., have reported that the $\mathrm{c} .3548 \mathrm{~A}>\mathrm{G}$ missense has high frequency in patients who were tested negative for BRCA1 and BRCA2 mutations in Algerian breast/ovarian cancer families (Cherbala et al., 2012).

We have found two unclassified intronic variants (IVS16-68 G>A, IVS16-92 G>A) that were reported twice or more in the studies. These two intronic mutations previously reported in Greek, Netherlands, Belgium, Argentina breast/ovarian cancer families and Singapore Malay women with early onset breast/ovarian cancer (Konstantopoulou et al., 2000; Sng et al., 2003). BRCA1 IVS16-68 G>A and IVS16-92 G>A have been reported previously in the BIC database, and they are among the top 20 mutation frequencies that have been described by 
designation (Sng et al., 2003).

Nevertheless, this meta-analysis also had some limitations. Firstly, the samples size for the association about BRCA $1 / 2$ and breast cancer common mutations and articles about Iranian populations are too small to provide strong information and more original studies are needed to further confirm our findings. Secondly, previous study only screened particular region of the BRCA1/2 genes might naturally exclude many potential common polymorphism in other region, so our results may not have sufficient statistical power to identify and analysis common polymorphism in the Iranian breast cancer patients.

Although there are some limitations in this metaanalysis, values of this study should also be highlighted. Firstly, as far as we know, this is the first meta-analysis to examine the common mutation of the BRCA genes in the Iranian breast cancer patients. Finally, any obvious evidence of publication bias could be detected in all genetic models.

In conclusion, this Meta-analysis results will be helpful to understand the possibility of the first true founder mutation of BRCA1/BRCA2 identified in the Iranian population, establish a genetic screening strategy, to provide individual risk assessment, and to design better therapeutic strategies in the Iranian population. However, the relatively high frequency of these mutations in the Iranian breast cancer patients cannot be explained by founder effects and It is possible that we have not yet identified all recurrent mutations that occur in Iran. Therefore, further population surveys of BRCA mutations will be necessary.

\section{Acknowledgements}

This research was supported by Shahid Sadoughi University of Medical Sciences, Yazd, Iran. The authors would like to thank Professor Seyed Mahdi Kalantar for impetus to perform this study.

\section{References}

Bar-Sade RB, Kruglikova A, Modan B, et al (1998). The 185delAG BRCA1 mutation originated before the dispersion of Jews in the diaspora and is not limited to Ashkenazim. Hum Mol Genet, 7, 801-5.

Begg CB, Mazumdar M (1994). Operating characteristics of a rank correlation test for publication bias. Biometrics, 50, 1088-101.

Cao YW, Fu XG, Wan GX, et al (2014). BRCA1 gene exon 11 mutations in Uighur and Han women with early-onset sporadic breast cancer in the northwest region of China. Asian Pac J Cancer Prev, 15, 4513-8.

Cherbal F, Salhi N, Bakour R, et al (2012). BRCA1 and BRCA2 unclassified variants and missense polymorphisms in Algerian breast/ovarian cancer families. Dis Markers, 32, 343-53.

Dey S, Soliman AS (2010). Cancer in the global health era: opportunities for the Middle East and Asia. Asia Pac J Pub Health, 22, 75-82.

Dombernowsky SL, Weischer M, Freiberg JJ et al (2009). Missense polymorphisms in BRCA1 and BRCA2 and risk of breast and ovarian cancer. Cancer Epidemiol Biomarkers
Prev, 18, 2339-41.

Easton DF (1999). How many more breast cancer predisposition genes are there? Breast Cancer Res, 1, 14-17.

Egger M et al (1997). Bias in meta-analysis detected by a simple, graphical test. $B M J, \mathbf{3 1 5}, 629-34$.

Fattahi MJ, Mojtahedi Z, Karimaghaee N, Talei A-R, Banani SJ, Ghaderi A (2009) Analysis of BRCA1 and BRCA2 mutations in southern Iranian breast cancer patients. Archives of Iranian Medicine, 12, 584-7.

Ghaderi A, Talei A, Farjadian S, et al (2001). Germline BRCA1 mutations in Iranian women with breast cancer. Cancer Lett, 165, 87-94.

Hansa J, Kannan R, Ghosh SK (2012). Screening of 185DelAG, 1014DelGT and 3889DelAG BRCA1 mutations in breast cancer patients from North-East India. Asian Pac J Cancer Prev, 13, 5871-4.

Haytural H, Yalcinkaya N, Akan G, (2013) Identification of a novel BRCA2 and CHEK2 A-C-G-C haplotype in Turkish patients affected with breast cancer. Asian Pac J Cancer Prev, 14, 3229-35.

Hasan TN, Shafi G, Syed NA, et al (2013). Lack of association of BRCA1 and BRCA2 variants with breast cancer in an ethnic population of Saudi Arabia, an emerging high-risk area. Asian Pac J Cancer Prev, 14, 5671-4.

Keshavarzi F, Noughani AE, Ayoubian M, Zeinali S (2012). Sequence variants of BRCA1 and BRCA2 genes in four Iranian families with breast and ovarian cancer. Iran J Public Health, 40, 57-66.

Keshavarzi F, Javadi GR, Zeinali S (2012). BRCA1 and BRCA2 germline mutations in 85 Iranian breast cancer patients. Familial Cancer, 11, 57-67.

Kooshyar MM, Nassiri M, Mahdavi M, Doosti M, Parizadeh A (2013). Identification of germline BRCA1 mutations among breast cancer families in Northeastern Iran. Asian Pac J Cancer Prev, 14, 4339-45.

Konstantopoulou I , Kroupis C, Ladopoulou A, et al (2000). BRCA1 mutation analysis in breast/ovarian cancer families from Greece. Hum Mutat, 16, 272-3.

Moslehi R, Kariminejad MH, Ghaffari V, Narod S (2003). Analysis of BRCA1 and BRCA2 mutations in an Iranian family with hereditary breast and ovarian cancer syndrome. American Journal of Medical Genetics, 117, 304-305.

Mehdipour P, Hosseini-Asl S, Savabi-E A, et al (2006). Low frequency of 185delAG founder mutation of BRCA1 gene in Iranian breast cancer patients. J Cancer Mol, 2, 123-7.

Medimegh I, Troudi W, Stambouli N, et al (2014). Wild-type genotypes of BRCA1 gene SNPs combined with microRNA over-expression in mammary tissue leading to familial breast cancer with an increased risk of distant metastases' occurrence. Med Oncol, 31, 255.

Miki Y, Swensen J, Shattuck-Eidens D, et al (1994). A strong candidate for the breast and ovarian cancer susceptibility gene BRCA1. Science, 266, 66-71

Pietschmann A, Mehdipour P, Mehdipour P, et al (2005). Mutation analysis of BRCA1 and BRCA2 genes in Iranian high risk breast cancer families. J Cancer Res Clin Oncol, 131, 552-8.

Pilato B, Martinucci M, Danza K, et al (2011). Mutations and polymorphic BRCA variants transmission in breast cancer familial members. Breast Cancer Res Treat, 125, 651-7.

Quintana-Murci L, Gal I, Bakhan T, et al (2005). The Tyr978X BRCA1 mutation, occurrence in non-Jewish Iranians and haplotype in French-Canadian and non-Ashkenazi Jews. Familial Cancer, 4, 85-88.

Rassi H, Houshmand M, Hashemi M, et al (2008). Application of multiplex PCR with histopathologic features for detection of familial breast cancer in formalin-fixed, paraffin-embedded 
Mohammad Forat-Yazdi et al

histologic specimens. Cytology and Genetics, 42, 55-62.

Rezaianzadeh A, Sepandi M, Akrami M, et al (2014). Pathological profile of patients with breast diseases in Shiraz. Asian Pac J Cancer Prev, 15, 8191-5.

Perez JAM, Garcia FC, Palacios S, et al (2009). Epidemiology of risk factors and symptoms associated with menopause in Spanish women. Maturitas, 62, 30-6.

Saleh gohari N, Mohammadi-Anaie M, Kalantari-Khandani B (2012) BRCA1 gene mutations in breast cancer patients from Kerman Province, Iran. Iranian J Cancer Prev, 5, 210-5.

Sepandi M, Akrami M, Tabatabaee H, Rajaeefard et al (2014). Breast cancer risk factors in women participating in a breast screening program, a study on 11,850 Iranian females. Asian Pac J Cancer Prev, 15, 8499-502.

Sng JH, Ali AB, Lee SC, et al (2003). BRCA1 c.2845insA is a recurring mutation with a founder effect in Singapore Malay women with early onset breast/ovarian cancer.J Med Genet, 40, 117.

Solano AR, Aceto GM, Delettieres D, et al (2012). BRCA1 and BRCA2 analysis of Argentinean breast/ovarian cancer patients selected for age and family history highlights a role for novel mutations of putative south-American origin. Springerplus, 1, 20.

Taghavi A, Fazeli Z, Vahedi M, et al (2012). Increased trend of breast cancer mortality in Iran. Asian Pac J Cancer Prev, 13, 367-70.

Welcsh PL, King MC (2001). BRCA1 and BRCA2 and the genetics of breast and ovarian cancer. Human Molecular Genetics, 10, 705-13.

Yassaee VR, Zeinali S, Harirchi I (2002). Novel mutations in the BRCA1 and BRCA2 genes in Iranian women with earlyonset breast cancer. Breast Cancer Res, 4, 6 . 